\title{
Critical Aspects of the Cracked Brazilian-disc test Explored by the Method of Caustics
}

\author{
Stavros K. Kourkoulis* \\ Department of Mechanics, School of Applied Mathematical and Physical Sciences, National Technical University of Athens, 5 Heroes of Polytechnion \\ Avenue, Theocaris Bld., Zografou Campus, 15773 Athens, Greece
}

Dr Stavros K. Kourkoulis was born in 1961. He graduated from the School of Mechanical Engineering of the National Technical University of Athens (NTUA) in 1984 and obtained his PhD from the Engineering Science Department of the same University in 1989 under the supervision of late Academician Professor P. S. Theocaris. Since 2009 he is Associate Professor at the School of Applied Mathematical and Physical Sciences of NTUA. He is the author of about eighty publications in peer-reviewed scientific journals and of about ninety publications in proceed-ings of international conferences with full-text review. He authored two text books on Strength of Materials (in Greek) and edited four volumes with conference proceedings. He is Scientific Responsible in twenty one research projects of total budget exceeding 2.5 million Euros. He has taught more than sixty semester-courses on Mechanics, Strength of Materials and Biomechanics. His main research interests are focused to the failure of materials, the mechanical behaviour of rock-like materials (with emphasis to materials used for the restoration and conservation of Cultural Heritage monuments) and the Biomechanics of the Musculoscetal System.

\begin{abstract}
Some critical aspects of the cracked Brazilian-disc test are explored in the light of recently introduced analytic solutions for the stress- and displacementfields developed in a centrally cracked cylindrical disc under uniform pressure (acting along two finite arcs of its periphery symmetric with respect to the disc's center). The study is focused to the reliable determination of the respective Stress Intensity Factors (SIFs) and also to the role of the actual boundary conditions along the discjaw contact arc (assuming that the disc is compressed between the curved jaws of the device suggested by the International Society for Rock Mechanics (ISRM) for the standardized implementation of the Brazilian-disc test). The crack is assumed relatively short with respect to the disc's radius. The results of the analytic approach concerning the SIFs and the length of the disc-jaw contact arc are compared to the respective ones obtained experimentally using the method of Caustics. The agreement is satisfactory at least for load levels for which the linearity assumption is not violated. Taking advantage of the insight gained additional issues related to the validity of the test as a mean to determine mode-I fracture toughness are critically discussed revealing that quite a few questions are still open although the test is standardized by ISRM almost since 20 years.
\end{abstract}

Keywords: Cracked Brazilian Disc, Rock-like Materials, Linear Elasticity, Complex Potentials, Contact arc, Closing Cracks, "Overlapping", Caustics.

\section{Introduction}

The Brazilian-disc test was introduced by Carneiro [1] and Akazawa [2] as a substi- 
tute of the direct tension test in case of specimens made of concrete or brittle geomaterials. Due to its simplicity the test became soon very popular and (in spite of quite a few concerns about the relation between the quantity obtained from this test and the actual tensile strength $[3,4]$ ) the respective procedure was standardized by ISRM already since 1978 [5].

Besides its use for the determination of the tensile strength the Brazilian-disc test is nowadays widely used also for the determination of mode-l fracture toughness KIC (assuming of course that the disc-shaped specimen is centrally cracked as it is shown in Fig. 1). This quantity, which somehow quantifies the resistance to crack initiation under tensile loading, is an irreplaceable tool for many engineering applications. Although the experimental procedure for the determination of KIC using the cracked Brazilian-disc test (in the form of the Cracked Chevron Notched Brazilian Disc - CCN$\mathrm{BD}$ ) is also standardized by ISRM since 1995 [6], a number of critical issues are still to be enlightened $[7,8]$. They are related to either practical laboratory aspects (i.e. machining central cracks in rock-like specimens, reducing friction at the disc-jaw interface etc) and also to the independence of the final outcome of the test from the geometry of the specimen. The latter is of crucial importance since it is well documented in the literature that using differently-shaped specimens the values obtained for $\mathrm{KIC}$ are not identical with those obtained using the standardized cracked Brazilian disc-test [9]. Moreover some researchers believe that "... the formula of ISRM was inadequate and inaccurate" [10] while even Fowell, who is among the founders of the respective ISRM standard stated, that "... it may be necessary to revise the dimensionless SIF values for a future release of the suggested method to incorporate some recent developments. More research and input from different sources need to be coordinated [11]".

To the present author's opinion the main reasons for the above mentioned inconsistencies are two: (i) the lack of a convincing theoretical model describing the displacement- and stress-fields developed in a cracked circular disc compressed between the curved jaws of the ISRM suggested device for the standardized realization of the Brazilian-disc test (which in turn results to insuperable difficulties prohibiting the determination of sound values of the respective SIFs) and (ii) the fact that the actual boundary conditions prevailing along the disc-jaw interface are ignored since it is usually assumed that their influence is limited in the immediate vicinity of the disc-jaw contact arc. Both problems are extremely complicated and closed form solutions are not to be expected especially when the length of the crack is comparable to the diameter of the disc.

In this context an attempt is here described to deal with these issues by taking advantage of recently introduced solutions for the stress- and displacement-fields in a centrally cracked circular disc (assuming that the crack is relatively short compared to the disc's radius) [12] and for the naturally accepted boundary conditions in case the Brazilian-disc test is considered as a contact problem of two elastic cylindrical bodies [13]. The solution in both cases was achieved by employing Kolosov's - Muskhelishvili's [14, 15] complex potentials technique.

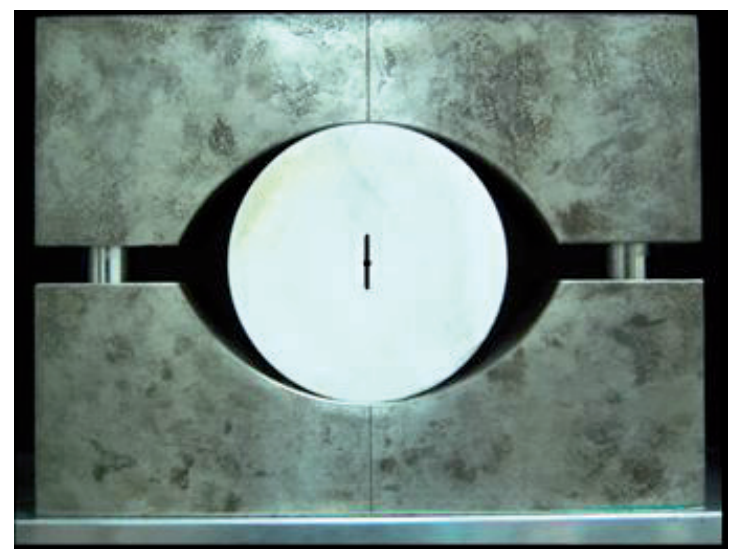

Fig. 1: The device suggested by ISRM for the standardized implementation of the Brazilian-disc test. A typical cracked disc shaped specimen with a central crack is placed between the jaws of the device with the crack oriented along the symmetry axis of the pressure distribution.

The formulae obtained for the contact arc and the load distribution along the contact arc as well as for the stresses and displacements (especially in the immediate vicinity of the crack tip along the crack axis) are relatively simple and compact.

As a first step the formulae for the stresses around the crack tip are used to obtain closed form expressions for the SIFs which are valid through the whole range of crack inclinations with respect to the loading axis, $\phi_{0}$, even for configurations for 
which the crack lips are coming in contact generating additional normal and tangential stresses (contact stresses) along the crack lips. The latter is a critical point and has been recognized by many authors as the source of quite a few misunderstandings. Atkinson et al. [16] in their pioneering work clearly stated that "at the point of crack closure, the model of the crack must be altered." In case crack closure is ignored the formal application of the theoretical formulae may lead to unnatural phenomena as it is for example the overlapping of the crack lips or their mutual interpenetration or the negative values for the mode-I SIF.

The validity of the results obtained is then assessed by comparing the SIFs-values with those experi-mentally obtained using the Reflected Caustics method. The same experimental tool is used to explore the validity of the solution concerning the length of the contact arc and the assumption about its equality with the respective quantity in case of intact discs. In both cases the agreement is satisfactory as long as the linearity assumption is satisfied.

Based on the insight gained from the analytic and experimental study some additional controversial aspects of the cracked Brazilian-disc test are discussed, leading to the conclusion that the procedure followed for the standardized determination of KIc should be further studied before its outcome is definitely accepted as the true value of fracture toughness.

\section{Theoretical Preliminaries \\ 2.1. Compact expressions for the SIFs in a circular disc with a short central crack}

A centrally cracked circular disc, of radius $\mathrm{R}$ and thickness $\mathrm{t}$, is subjected to a uniformly distributed radial pressure $\mathrm{p}$ along two symmetric arcs of its periphery L (Fig. 2). The length of the crack, $2 \alpha$, is relatively small compared to the disc's radius. The axis of symmetry of the pressure $p$ forms an angle $\phi_{0}$ with respect to the axis of the crack. The end points of the loaded rims are denoted as $t_{j}$, $\mathrm{j}=1,2,3,4$. The disc is in the $\mathrm{z}=\mathrm{x}+\mathrm{iy}=\mathrm{re}^{\mathrm{i} \vartheta}$ complex plane with the origin of the Cartesian reference system at the disc's centre. The crack is oriented along the $x$-axis. The problem is considered as a plane one and the material as homogeneous and isotropic.

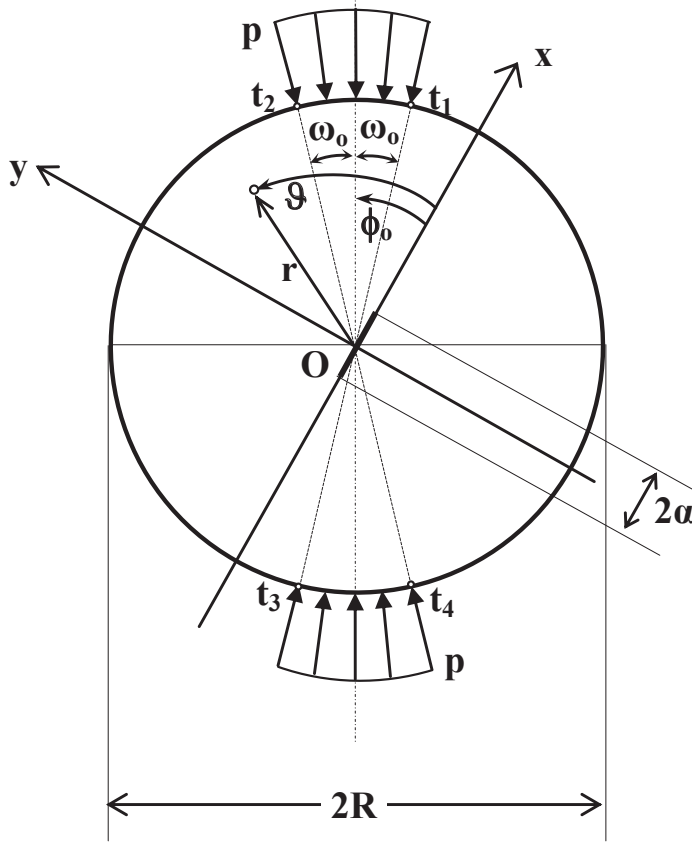

Fig. 2: The mathematical problem and the definition of symbols.

The complex potentials characterizing the elastic equilibrium of the as above geometry were recently [12] obtained in the form:

$$
\begin{aligned}
& \left.\begin{array}{l}
\Phi(z) \\
\Omega(z)
\end{array}\right\}=\frac{p}{4 \pi i}\left[\frac{z}{\sqrt{z^{2}-\alpha^{2}}}\left(4 i \omega_{o}-\ln \frac{t_{2}^{2}-z^{2}}{t_{1}^{2}-z^{2}}+\ln \frac{\overline{t_{2}^{2}}-z^{2}}{\overline{t_{1}^{2}}-z^{2}}\right) \mp\right. \\
& \left.\left(\ln \frac{t_{2}^{2}-z^{2}}{t_{1}^{2}-z^{2}}+\ln \frac{\overline{t_{2}^{2}}-z^{2}}{\overline{t_{1}^{2}}-z^{2}}\right)+2\left(z^{2}-R^{2}\right)\left(\frac{z}{\sqrt{z^{2}-\alpha^{2}}} \mp 1\right)\left(\frac{1}{\overline{t_{1}^{2}}-z^{2}}-\frac{1}{\overline{t_{2}^{2}}-z^{2}}\right)\right]
\end{aligned}
$$

Knowing the complex potentials it is possible (following Muskhelishvili's [15] technique) to determine the stress components at any point of the disc. Focusing attention to the linear locus along the crack axis (excluding the crack itself) in the immediate vicinity of the crack tips the stress field is given as [12]: 


$$
\begin{aligned}
& \underset{\substack{\sigma_{x} \\
x \rightarrow|\alpha|}}{ }=\left[\delta \frac{(\kappa+1)(3-\kappa)}{2 \kappa}-4\right] \frac{P_{\text {frame }}}{2 \pi R \omega_{o} t} \cos 2 \phi_{o} \sin 2 \omega_{o}-\delta \frac{(\kappa+1)(3-\kappa) P_{\text {frame }}}{4 \kappa \pi R t}- \\
& {\left[\delta \frac{(\kappa+1)(3-\kappa)}{2 \kappa}-2\right] \frac{P_{\text {frame }}}{2 \pi R t}\left(\frac{\cos 2 \phi_{o} \sin 2 \omega_{o}}{\omega_{o}}-1\right) \frac{|x|}{\sqrt{x^{2}-\alpha^{2}}}} \\
& \underset{x \rightarrow|\alpha|}{\sigma_{y}}=\delta \frac{(\kappa+1)^{2} P_{\text {frame }}}{4 \kappa \pi R t}\left(\frac{\cos 2 \phi_{o} \sin 2 \omega_{o}}{\omega_{o}}-1\right)-\left[\delta \frac{(\kappa+1)^{2}}{2 \kappa}-2\right] \frac{P_{\text {frame }}}{2 \pi R t} . \\
& \left(\frac{\cos 2 \phi_{o} \sin 2 \omega_{o}}{\omega_{o}}-1\right) \frac{|x|}{\sqrt{x^{2}-\alpha^{2}}} \\
& \underset{x \rightarrow|\alpha|}{\sigma_{x y}}=-\tau \frac{(\kappa+1)^{2} P_{\text {frame }}}{4 \kappa \pi R \omega_{o} t} \sin 2 \phi_{o} \sin 2 \omega_{o}+\left[\tau \frac{(\kappa+1)^{2}}{2 \kappa}-2\right] \frac{P_{\text {frame }}}{2 \pi R \omega_{o} t} . \\
& \sin 2 \phi_{o} \sin 2 \omega_{o} \frac{|x|}{\sqrt{x^{2}-\alpha^{2}}}
\end{aligned}
$$

where: $\kappa$ is Muskhelishvili's constant and $\mathrm{P}_{\text {frame }}=2 \mathrm{pR} \omega_{\mathrm{o}}$ is the overall external load exerted to the disc by the loading frame. Concerning parameters $\tau$ and $\delta$ they are of importance only in case the crack inclination angle $\phi_{0}$ exceeds the critical value $\phi_{\text {cr }}$ which corresponds to cracks the lips of which are coming in mutual contact and therefore contact stresses are generated altering the initial boundary conditions $[12,16,17]$. In the case considered here $\left(\phi_{0}<\phi_{\text {cr }}=0\right)$ it holds that $\tau=\delta=0$. As far as it concerns the contact arc $\omega_{\mathrm{o}}$ it is here assumed that it is given by the respective formula for the intact Brazilian disc [18], since the crack is considered "short" with respect to the disc's diameter (the accuracy of the specific assumption will be checked experimentally in Section 3).
$\omega_{o}=\operatorname{Arcsin} \sqrt{\frac{6 P_{\text {frame }}}{\pi R t}\left(\frac{\kappa+1}{4 \mu}+\frac{\kappa_{J}+1}{4 \mu_{J}}\right)}(4)$

In Eq. $(4)(\kappa, \mu)$ and $(\kappa \mathrm{J}, \mu \mathrm{J})$ are Muskhelishvili's constant and shear modulus of the disc's and jaw's materials, respectively. The jaw's radius of curvature, according to the ISRM standard, is assumed equal to $1.5 R$ [5].

Given the stress field along the crack axis one can easily obtain the mode-I and mode-II SIFs. Especially for crack inclinations which do not correspond to geometries with contact of their lips (i.e. $\tau=\delta=0$ ) the SIFs (adopting the traditional representation) read as:

$$
\begin{aligned}
& K_{I}=\frac{P_{\text {frame }}}{R t} \sqrt{\frac{\alpha}{\pi}} \cdot \underbrace{\left[\frac{\cos 2 \phi_{o} \sin 2 \omega_{o}-\rho^{2} \sin 4 \omega_{o}}{\omega_{o}\left(1-4 \rho^{2} \cos 2 \phi_{o} \cos 2 \omega_{o}\right)}-1\right]}_{F_{I}\left(\phi_{\left.\phi_{o}, \omega_{o}, \delta\right)}\right.} \\
& K_{I I}=\frac{P_{\text {frame }}}{R t} \sqrt{\frac{\alpha}{\pi}} \cdot \underbrace{\frac{-\sin 2 \phi_{o} \sin 2 \omega_{o}}{\omega_{o}\left(1-4 \rho^{2} \cos 2 \phi_{o} \cos 2 \omega_{o}\right)}}_{F_{I I}\left(\rho, \phi_{o}, \omega_{o}, \tau\right)}
\end{aligned}
$$

\subsection{The reflected caustics technique as applied for the de- termination of SIFs and the contact length}

Shortly after Manogg introduced the method of Transmitted Caustics $[19,20]$ the application of which demands specimens made of transparent materials, Theocaris presented the Reflected Caus- tics method permitting the study also of opaque materials [21, 22]. Both methods are based on simple principles of Geometric Optics: In case a light beam impinges on a specimen at the vicinity of an intense stress field the light rays (received on a reference plane parallel to the specimen) will 
concentrate along a strongly illuminated curve, the caustic, due to the strong thickness variations of the specimen. The shape and size of the caustic curve permit quantitative investigation of critical features of the stress field and the geometry of the deformed area. The former property is used for the determination of SIFs while the latter is used for the quantification of the contact length.

In case of cracks under combined compressive and shear loading (which is the case of the cracked Brazilian disc with $\phi_{0} \neq 0$ ) Theocaris and Sakellariou [23] assuming traction-free crack lips (in other words for $\left.\phi_{0}<\phi_{\text {cr }}\right)$ proved that the mode-I and mode-II SIFs are given as:

$$
K_{I}=\frac{0.311\left[D_{(-)}\right]^{\frac{5}{2}}}{Z_{o} t c \lambda_{\Perp}^{\frac{3}{2}}}, K_{I I}=K_{I} \tan \left(\phi_{o}\right)
$$

where: $\mathrm{D}_{(-)}$is the diameter of the caustic in the compressive field, $\mathrm{t}$ is the specimen's thickness, $\mathrm{Z}_{\mathrm{o}}$ is the distance between the specimen and the screen, $\mathrm{c}$ is the optical constant of the plate for reflections of the light rays from the rear face of the plate and $\lambda_{\mathrm{m}}$ is the magnification factor of the setup (depending on the distances of the focus of the light bundle and of the screen from the ring's front face). It is important to note here that Theocaris and Petrou [24] proved that the $\mathrm{K}_{\mathrm{I}}$ - values calculat- ed from caustics at the extremities of slits (artificial cracks in the form of rectangular hole) with a side ratio $m$ exceeding 40, differ from the corresponding values of the Griffith crack by less than $5 \%$. This conclusion is very important for the present study since the cracks machined in the experimental protocol are slits with $\mathrm{m}=40$ and $\mathrm{m}=80$ rather than Griffith cracks.

A few years later Theocaris and Stassinakis [25] used the method of Reflected Caustics to explore also contact problems. Their approach was recently improved by taking into account also the in-plane displacement field [26] which was ignored in the initial formulation. It was proven that the contact length, $\ell$, developed in case a circular disc or a ring (with a relatively small radius of its inner circle) is compressed between the jaws of the ISRM device is given by the following expression (Fig. 3) [26]:

$\ell^{2}+2\left|\mathrm{C}^{*}\right|^{\frac{2}{3}} \ell^{\frac{4}{3}}-\frac{\left[2 \mathrm{~W}_{\mathrm{x}^{\prime}}\left(\alpha_{\mathrm{d}}\right)\right]^{4}}{4 \lambda_{\mathrm{m}}^{2}}=0$

$\mathrm{C}^{*}$ is a constant depending on the ring's material, the geometry and the experimental set-up, $W_{x^{\prime}}$ is the $x$-Cartesian component of the vector $\vec{W}$ describing the caustic curve and $\alpha_{\mathrm{d}}$ is the initial curve's, Ad, end-point (Fig. 3). According to the analysis thoroughly described in ref. [26] $\mathrm{W}_{x^{\prime}}$ is given as:

$$
\begin{aligned}
& \mathrm{W}_{\mathrm{x}^{\prime}}=\lambda_{\mathrm{m}}\left[\mathrm{r}_{\mathrm{o}} \cos \vartheta-\mathrm{r}_{\mathrm{o}}\left(\frac{2\left|\mathrm{C}^{*}\right|}{\ell}\right)^{\frac{2}{3}} \sin \left(\vartheta-\frac{\vartheta_{1}+\vartheta_{2}}{2}\right)\right]+\frac{\lambda_{\mathrm{m}}}{24 \mu \mathrm{R}_{\mathrm{O}} \mathrm{K}}\left\{( \kappa - 1 ) \left[\mathrm{r}_{\mathrm{o}}^{2} \sin 2 \vartheta-\right.\right. \\
& \mathrm{r}_{\mathrm{o}}\left(2\left|\mathrm{C}^{*}\right|\right)^{\frac{1}{3}} \ell^{\frac{2}{3}} \sin \left(\vartheta+\frac{\vartheta_{1}+\vartheta_{2}}{2}\right)+\frac{\ell^{2}}{2}\left(\tan ^{-1} \frac{\mathrm{r}_{\mathrm{o}} \sin \vartheta+\left(2\left|\mathrm{C}^{*}\right|\right)^{\frac{1}{3}} \ell^{\frac{2}{3}} \sin \frac{\vartheta_{1}+\vartheta_{2}}{2}}{\mathrm{r}_{\mathrm{o}} \cos \vartheta+\left(2\left|\mathrm{C}^{*}\right|\right)^{\frac{1}{3}} \frac{\ell^{\frac{2}{3}} \cos \frac{\vartheta_{1}+\vartheta_{2}}{2}}{2}}\right. \\
& \left.\left.\left.\tan ^{-1} \frac{\mathrm{r}_{\mathrm{o}} \sin \vartheta-\left(2\left|\mathrm{C}^{*}\right|\right)^{\frac{1}{3}} \ell^{\frac{2}{3}} \sin \frac{\vartheta_{1}+\vartheta_{2}}{2}}{\mathrm{r}_{\mathrm{o}} \cos \vartheta-\left(2\left|\mathrm{C}^{*}\right|\right)^{\frac{1}{3}} \ell^{\frac{2}{3}} \cos \frac{\vartheta_{1}+\vartheta_{2}}{2}}\right)\right]+4 \mathrm{r}_{\mathrm{o}} \sin \vartheta\left[\mathrm{r}_{\mathrm{o}} \cos \vartheta-\left(2\left|\mathrm{C}^{*}\right|\right)^{\frac{1}{3}} \ell^{\frac{2}{3}} \cos \frac{\vartheta_{1}+\vartheta_{2}}{2}\right]\right\}
\end{aligned}
$$




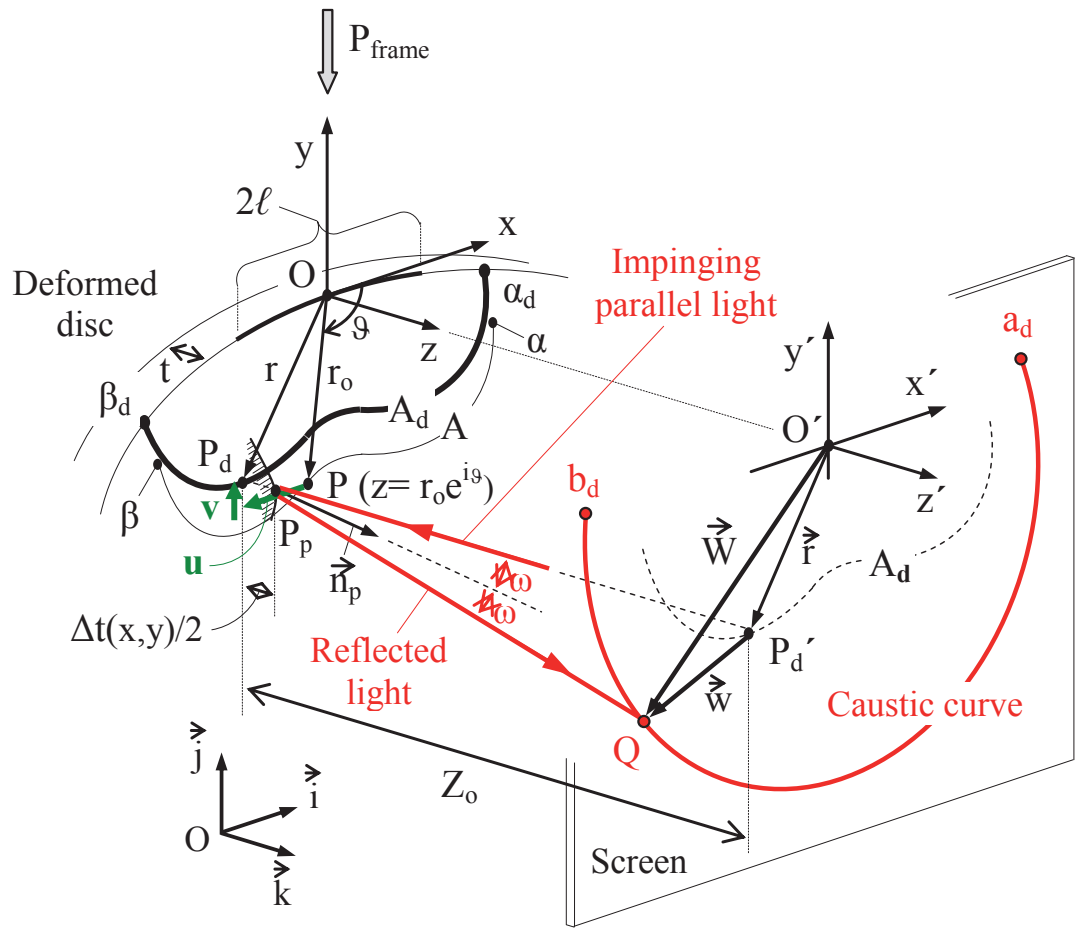

Fig. 3: The basic principles of the Reflected Caustics method and definition of symbols in case the method is used for the determination of the contact length.

In the above Eq.(8) ro is the radius of a point $z$ on the initial curve (Fig. 3) while angles $\vartheta_{1}, \vartheta_{2}$ and radii $\mathrm{r}, \mathrm{r}_{1}, \mathrm{r}_{2}$ are defined in Fig. 4 .

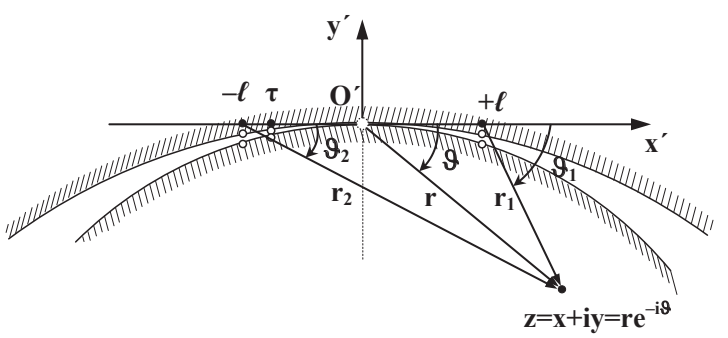

Fig. 4: Definition of symbols appearing in Eq.(8).

\section{Experimental Procedure and Results}

Cylindrical discs, of radius $\mathrm{R}=10 \mathrm{~cm}$ and thickness $\mathrm{t}=1 \mathrm{~cm}$, made of PMMA were compressed between the jaws of the ISRM suggested device for the standardized realization of the Braziliandisc test. Central cracks were machined using a $0.25 \mathrm{~mm}$ thick jigsaw driven through a pilot hole of diameter $0.5 \mathrm{~mm}$. Two crack lengths were considered equal to either 10 or $20 \mathrm{~mm}$. For the crack inclination angle, $\phi_{0}$, three cases were tested: $\phi_{0}=0^{\circ}$ (pure mode-I), $\phi_{0}=10^{\circ}$ (mixed mode), and $\phi_{0}=30^{\circ}$ (according to the analytic solution it corresponds to pure mode-II).

The typical experimental set-up of the Caustics method was used [21], shown schematically in Fig. 5a. The ISRM device was mounted to a $50 \mathrm{kN}$ electromechanical INSTRON loading frame. An overall view of the experimental set-up is shown in Fig. 5b. The experiments were carried out under displacement-control mode at a rate of $0.2 \mathrm{~mm} / \mathrm{min}$. It is mentioned that this value is not in agreement with the demands of the respective standards [5, 6] which dictate either a predefined stress intensity rate (below $0.25 \mathrm{MPa} \sqrt{\mathrm{m}} / \mathrm{sec}$ ) or a predefined test duration ranging from 10 to $20 \mathrm{sec}$. However these tests speeds are unconstructive for the purposes of the present protocol and therefore the rate of $0.2 \mathrm{~mm} / \mathrm{min}$ was qualified, providing monotonic test conditions. In any case it is not expected that speed differences of this magnitude will significantly influence the results of the tests. The force induced was measured by a $50 \mathrm{kN}$ calibrated load cell. 

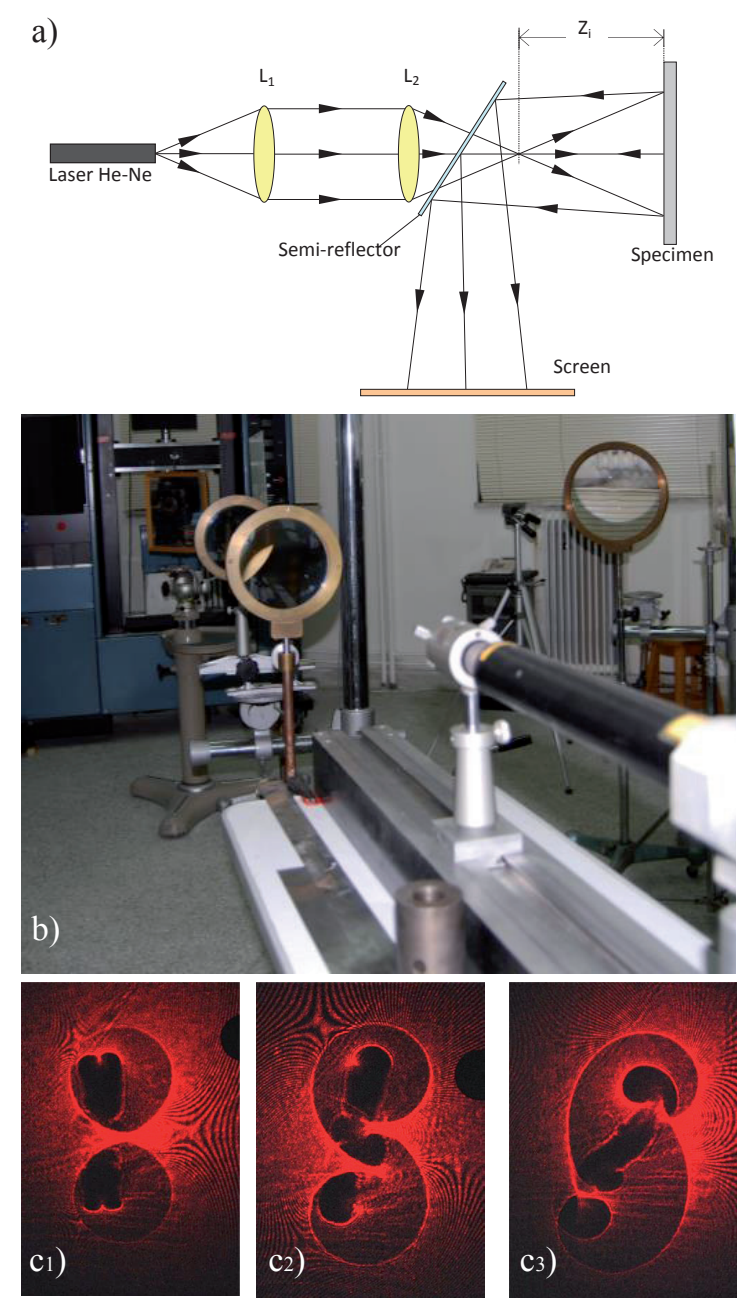

Fig. 5: The experimental set-up. Schematic representation (a) and a characteristic photo (b). Representative caustics for $\phi_{0}=0^{\circ}$ (c1), $\phi_{o}=10^{\circ}(\mathrm{c} 2)$ and $\phi_{o}=30^{\circ}$ (c3).

The caustics formed on the screen were photographed (some representative photos are shown in Figs. 5c) and their geometric characteristics were measured with the aid of a standard optical arrangement and suitable commercial software. Using these data and taking advantage of the formulae given in Section 2.2 the mode-I and modeII SIFs were calculated as functions of the load.

The experimental results are plotted in Fig. 6 together with the respective ones obtained from Eqs.(5). In this figure solid lines correspond to the theoretical predictions while square and rhomboid symbols represent the experimental data. Moreover filled symbols correspond to the experimental data for KI while the empty ones to the
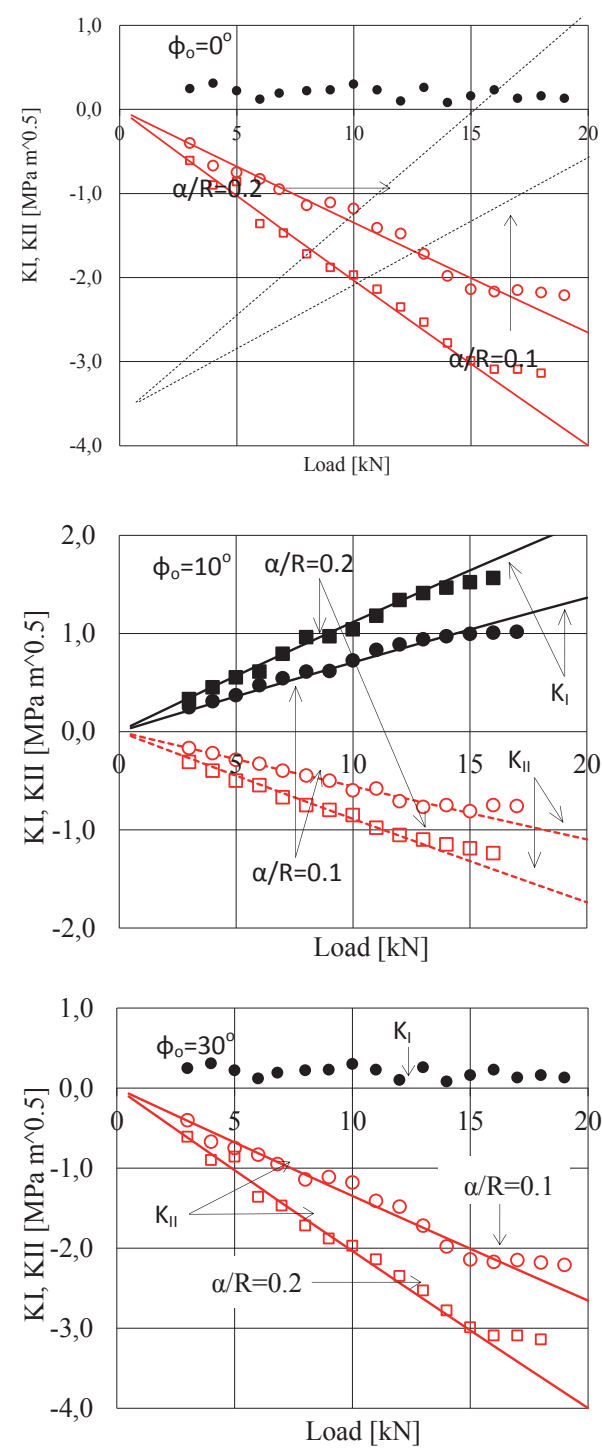

Fig. 6: Experimental versus analytic results for the mode-l and mode-II SIFs.

respective data for KII. It is seen from Fig. 6 that for low load levels the agreement is satisfactory for all cases. As the load increases some discrepancies appear which should be attributed to the violation of the linearity assumption in the immediate vicinity of the crack tip due to the strong local stress concentration. Moreover it should be noted that the dependence of the theoretically obtained SIFs on the externally applied load is not linear as it is expected according to the standard definition of these quantities (see for example the dotted lines in 

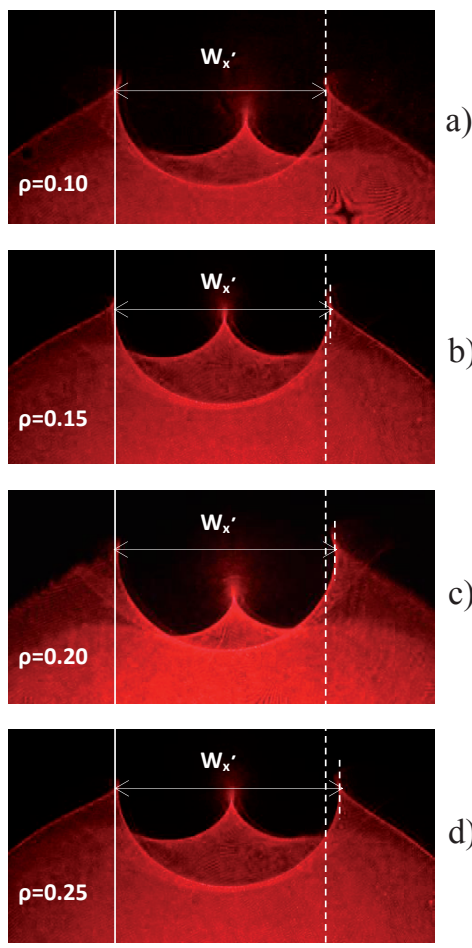

Fig. 7: The caustic curves formed due to the intensive stress field in the immediate vicinity of the disc-jaw contact arc. The characteristic quantity $W x^{\prime}$ related to the contact length is indicated.

the disc-jaw contact arc. The characteristic quantity $W x^{\prime}$ related to the contact length is indicated the first of the plots of Fig. 6). This deviation is due to the fact that the contact angle, $\omega_{0}$, changes as the load increases according to Eq.(4). Finally it is to be emphasized that for $\phi_{\mathrm{o}}=30^{\circ}$ the stress state does not correspond to pure mode-I since $\mathrm{K}_{\mathrm{I}}$ (although very small) is not zeroed (in fact for the specific configuration the critical angle, $\phi_{\mathrm{cr}}$, is equal to $29.3^{\circ}$ depending slightly on the crack length and angle $\omega_{0}$ ). This is mainly due to the fact that the cracks machined in the experimental protocol are in fact rectangular slits rather than mathematical cracks.

During the same experimental protocol the caustics formed due to the intensive strain field around the disc-jaw contact arc were also photographed. A typical series of such photos are seen in Fig. 7 for $\phi_{0}=0^{\circ}$ and crack length ratios equal to $\rho=0.10$, $0.15,0.20$ and 0.25 . It is seen that the characteristic quantity $\mathrm{W}_{\mathrm{x}^{\prime}}$, which according to Eq.7 determines the contact semi-length $\ell$, is more or less insensitive to the crack length as long as $\rho<0.20$. From this limit on the quantity $\mathrm{W}_{\mathrm{x}^{\prime}}$ starts increasing (see Fig.7d) indicating a decreased stiffness of the discjaw elastic system, obviously due to the increased length of the crack. From a quantitative point of view, in the range $0<0.2$, the relative error is well below $5 \%$ or in other words within the experimental accuracy (taking into account also the difficulties in experimentally quantifying the parameter $\left.\mathrm{W}_{x^{\prime}}[25,26]\right)$.

\section{Discussion}

Besides the points highlighted in previous sections attention should be also paid to some additional issues related to the cracked Brazilian-disc test. The need of redefining the concept of SIFs at least in case they are obtained from a mixed fundamental problem is perhaps the most crucial among them. Indeed as it is seen from Eqs.(5) both $\mathrm{KI}^{-}$and $\mathrm{K}_{\mathrm{II}}$-SIFs appear depending on material properties through the parameter $\omega_{\text {o, }}$ i.e. through the length of the contact arc. Such dependence is at least peculiar and further study is required in the direction of removing this material dependence, as it is done for example by Markides et al. [27]. It is mentioned however that the conclusion concerning this material dependence of the SIFs is not novel. Similar observations were already made by other researchers (see for example Hills et al. [28]). Along the same line Asfar and Ahmed [29] in their study about the SIFs for a pair of edge cracks in a semi-infinite medium with a distribution of eigenstrain and subjected to a far field uniform applied load concluded that the mode-I and mode-II SIFs are given as:

$$
K_{I, I I}=\sqrt{\pi \alpha} \frac{2 \mu}{\kappa+1} \sqrt{2} \varphi_{x, y}(+1)
$$

where: $\mu$ is the shear modulus and $\kappa$ is Kolosov's constant which is also a function of Poisson's ratio. The similarity of Eq.(9) with Eq.(5) is striking.

Another aspect of the test that should be carefully considered is the local distortion of the stress field induced by the tangential friction stresses inevitably developed along the disc-jaw interface. These stresses in the most general case of partial slip along the contact arc, in case a compact disc is squeezed between the jaws of the ISRM device, are described by the following equation [30]: 


$$
\left.\left.\left.\tan ^{-1} \frac{r_{0} \sin \vartheta-\left(2\left|C^{*}\right|\right)^{\frac{1}{3}} \ell^{\frac{2}{3}} \sin \frac{\vartheta_{1}+\vartheta_{2}}{2}}{r_{0} \cos \vartheta-\left(2\left|C^{*}\right|\right)^{\frac{1}{3}} \ell^{\frac{2}{3}} \cos \frac{\vartheta_{1}+\vartheta_{2}}{2}}\right)\right]+4 r_{0} \sin \vartheta\left[r_{0} \cos \vartheta-\left(2\left|C^{*}\right|\right)^{\frac{1}{3}} \ell^{\frac{2}{3}} \cos \frac{\vartheta_{1}+\vartheta_{2}}{2}\right]\right\}
$$

where: $\tau$ is any point with $\tau \in[-\ell,+\ell], K=\frac{\kappa+1}{4 \mu}+\frac{\kappa_{i}+1}{4 \mu_{j}}$. In addition $f$ is a real positive constant (units $\mathrm{m}^{-1}$ ) depending on the nature of the disc's and jaw's materials in contact, $\mathrm{n}$ is the familiar Amonton'sCoulomb's coefficient of dry friction and finally $\tau_{\text {crit }}$ denotes the point within the contact arc where slip starts. Taking advantage of Eq.(10) it can be proven that the relative deformability of the disc's and jaw's materials, as it is expressed by the ratio of their elastic moduli, is a decisive factor which governs the displacement mismatch along the discjaw interface and therefore it dictates the magnitude of the friction stresses developed [30]. This is clearly seen in Fig. 8 where the maximum value of the distribution of friction stresses is plotted against the ratio of the disc's over jaw's elastic moduli: Friction stresses approaching $250 \mathrm{MPa}$ could be generated in case of discs made of materials with Young's modulus approaching $50 \%$ of the respective modulus of steel (the material of the jaw). As a result it is concluded that in case of geomaterials with increased stiffness, comparable with that of the jaw's material, it is quite possible that the friction stresses cause premature fractures (either in the form of micro- or macro-cracking) in the immediate vicinity of the disc-jaw interface, jeopardizing seriously the validity of the test results regarding the fracture toughness.

The length and orientation of the crack is the last point that should be considered. It is already pointed out by many authors [31] that, in order for the final outcome to be valid, the length of the crack (in case the CCNBD test is used to determine the fracture toughness) should exceed a critical limit equal to about $50 \%$ of the disc's diameter. Clearly the formulae for the SIFs obtained in the present study are not applicable in such a case since the crack cannot be considered as a "short" one. Preliminary results from an approximate analytic solution for the problem of a disc with a relatively long central elliptic crack indicate that the results for the SIFs are significantly differentiated. This observation in conjunction with the respective one concerning

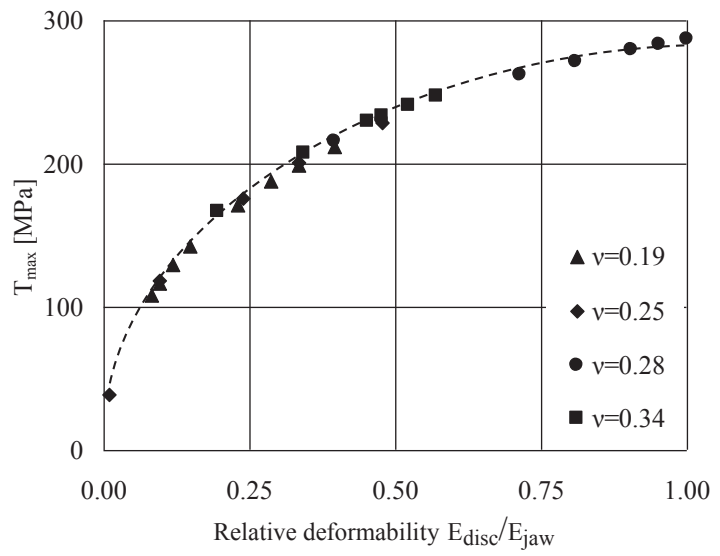

Fig. 8: The dependence of the friction stresses on the relative stiffness of the disc and jaw materials for various values of Poisson's ratio.

the rotation of the crack axis during loading [18] support further the view that the standardized formulae for Kic needs to be updated, as it has been already indicated by a number of researchers [7$11]$.

\section{Conclusion}

Some critical aspects of the cracked Braziliandisc test were considered in the light of recently published solutions for the stress- and displacement fields in a centrally cracked cylindrical disc under uniform pressure along two finite arcs of its periphery. It was concluded that, in case the crack is short compared to the disc's diameter (and also it is of mathematical nature, i.e. of zero distance between its lips), convenient compact expressions can be obtained for the SIFs, independently of whether the crack lips are stress free or in contact in the presence of contact stresses. In addition it was concluded that the length of the contact arc between the disc and the ISRM jaws is accurately enough approximated by the respective contact length of a compact disc compressed between the same jaws. The latter relieves existing models and analytic solutions from the need to adopt an 
arbitrary contact length (very "small" as it is usually stated) or even configurations with point forces.

Clearly the above points, as well as these discussed in Section 4, are extremely complicated and it is not to be expected that definite analytic answers could be given, especially for finite domains. Indeed while quite a few publications exist concerning, for example, the overlapping lips for a crack in an infinite medium [32-35] the respective ones for domains of finite dimensions are very rare. On the other hand, however, the rapid development of technology broadens the limits of experimental investigation of the respective topics improving also their accuracy and sensitivity. For example using the Digital Image Correlation Technique it was recently made possible to quantify the crack axis rotation [18]. The same as above is true for the development of engineering software and computational facilities which are nowadays irreplaceable tools in the hands of engineers. In this context the present study, in spite of the many simplifying assumptions adopted, could offer valuable help in the direction of validating and verifying such computational tools.

In any case it is evident that the cracked Brazilian-disc test is by no means closed. Additional focused research is required for the credibility of the standardized KIC determination with the aid of cracked cylindrical discs under compression (either according to ISRM or ASTM [36]) to be definitely established. Such a target is well beyond the scope of this paper and hopefully it will be the goal of in-depth team work coordinated by international societies like ISRM and ASTM.

\section{Acknowledgments}

The core of this paper was presented during the "30th Danubia-Adria Symposium on Advances in Experimental Mechanics", held at Primosten, Croatia, from 25th to 28th of September, 2013, under the auspices of the "Croatian Academy of Science and Arts". The assistance of Dr Ch.F. Markides in the ana-lytic formulation of the solution for the SIFs and the contribution of Professor D. N. Pazis of the Department of Mechanics of NTUA and Mr G. Bakalis during the execution of the reflected caustics experiments is kindly acknowledged.

\section{References}

[1] Carneiro, FLLB (1943). A new method to determine the ten- sile strength of concrete in Portuguese). Proce-7ed-ings of the 5th Meeting of the Brazilian Association for Technical Rules, 3d. Section, 126-129.

[2] Akazawa, T (1943). New test method for evaluating internal stress due to compression of concrete (the splitting tension test) Part 1. Journal of Japan Society of Civil Engineers, 29, 777-787.

[3] Fairhurst, C (1964). On the validity of the 'Brazilian' test for brittle materials. International Journal of Rock Mechanics and Mining Sciences, 1, 535-546.

[4] Mellor, M, Hawkes, I (1971). Measurement of tensile strength by diametral compression of discs and annuli. Engineering Geology, 5, 173-225.

[5] ISRM (1978). Suggested methods for determining tensile strength of rock materials. International Journal of Rock Mechanics and Mining Sciences, 15, 99-103.

[6] ISRM (Coordinator Fowell, RJ) (1995). Suggested methods for determining mode-i fracture toughness using CCNBD specimens, International Journal of Rock Mechanics and Mining Sciences, 32, 57-64.

[7] Iqbal, MJ, Mohanty, B (2006). Experimental calibration of stress intensity factor of the ISRM suggested CCNBD specimen used for the determination of mode-l fracture toughness. International Journal of Rock Mechanics and Mining Sciences, 43, 57-64.

[8] Iqbal, MJ, Mohanty, B (2007). Experimental calibration of ISRM suggested fracture toughness measure $\neg$ ment techniques in selected brittle rocks. Rock Mechanics and Rock Engineering, 40, 453-475.

[9] Dwivedi, RD, Soni, AK, Goell, RK, Dube, AK (2000). Fracture

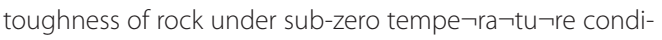
tions. International Journal of Rock Mechanics and Mining Sciences, 37, 1267-1275.

[10] Wang, QZ, Fan, H, Gou, XP, Zhang, S, (2013). Recalibration and clarification of the formula applied to the ISRM-suggested CCNBD specimens for testing rock fracture toughness. Rock Mechanics and Rock Engi-nne-7er-ing, 46, 303313.

[11] Fowell, RJ, Xu, C, Dowd, PA (2006). An update on the fracture toughness testing methods related to cracked CCNBD specimen. Pure and Applied Geophysics, 163, 1046-1057.

[12] Markides, ChF, Pazis, DN, Kourkoulis, SK (2013). The centrally cracked brazilian disc: Closed solutions for stresses and displacements for cracks under opening mode. Journal of Engineering Mathematics, 83, 143-168.

[13] Markides, ChF, Kourkoulis, SK (2013). Naturally accepted boundary conditions for the Brazilian disc test and the corresponding stress field. Rock Mechanics and Rock Engineering, 46, 959-980.

[14] Kolosov, GV (1935). Application of the Complex Variable to 
the Theory of Elasticity (in Russian). ONT1, Moscow-Leningrad.

[15] Muskhelishvili, NI (1963). Some Basic Problems of the Mathematical Theory of Elasticity. Noord $\neg$-hoff, Groningen, The Netherlands,

[16] Atkinson, C, Smelser, RE, Sanchez, J (1982). Combined mode fracture via the cracked Brazilian disk test. International Journal of Fracture, 18, 279-291.

[17] Pazis DN, Markides ChF, Kourkoulis SK (2013). The Brazilian disc with a short central crack under closing mode. Eproceedings of the 10th HSTAM International Congress on Mechanics, ISBN 124-7485-78969-567-3, 1-8.

[18] Kourkoulis, SK, Markides, ChF, Chatzistergos, PE (2012). The standardized Brazilian disc test as a contact problem. International Journal of Rock Mechanics and Mining Sciences, $57,132-141$.

[19] Manogg, P (1964). Anwendung der Schattenoptik zur Untersuchung des Zerreissvongangs von Platten. PhD Dissertation, Freiburg, Germany.

[20] Manogg, P (1966). Investigation of the rupture of a Plexiglas plate by means of an optical method involving high speed filming of the shadows originating around holes drilled in the plate. International Journal of Frac $\neg$ ture, 2, 604-613.

[21] Theocaris, PS (1970). Local yielding around a crack tip in Plexiglas. Journal of Applied Mechanics, 37, 409-415.

[22] Theocaris, PS, Gdoutos, EE (1972). An optical method for determining opening-mode and edge sliding-mode stress intensity factors. Journal of Applied Mechanics 39, 91-97.

[23] Theocaris, PS, Sakellariou, M (1990). Cracks and slits in compression and shear: An experimental study. Acta Mechanica, 85, 55-70.

[24] Theocaris, PS, Petrou, L (1989). From the rectangular hole to ideal crack. international journal of solids and structures, 25 , 213-233.

[25] Theocaris, PS, Stassinakis CA (1978). The elastic contact of two disks by the method of caustics. Experiᄀmental Mechanics, 18, 409-415.

[26] Kourkoulis, SK, Markides, ChF, Bakalis, G (2013). Smooth elastic contact of cylinders by caustics: The contact length in the Brazilian-disc test. Archives of Mechanics, 65, 313-338.

[27] Markides, ChF, Pazis, DN, Kourkoulis, SK (2011). Stress intensity factors for the brazilian disc with a short central crack: Opening versus closing cracks. Applied Mathematical Modelling, 35, 5636-5651.

[28] Hills, DA, Kelly, PA, Dai, DN, Korsunsky, AM (1996). Distributed dislocation fundamentals. Gladwell GML (editor), Solution of crack problems - The distributed dislocation technique. Kluwer Academic Punlishers, Dordrecht, Boston, London.

[29] Afsar, AM, Ahmed, SR (2006). Analysis of stress intensity fac- tors for a pair of edge cracks in semi-infinite medium with distributed eigenstrain. International Journal of Applied Mechanics and Engineering, 11, 269-287.

30] Kourkoulis, SK, Markides, ChF, Hemsley, JA (2013). Frictional stresses at the disc-jaw interface during the standardized execution of the Brazilian disc test. Acta Mechanica, 224, 255-268.

[31] Dong, S (2008). Theoretical analysis of the effects of relative crack length and to ading angle on the expe-ᄀri-mental results for crackedisraziliand disc testing. Engineering Fracture Mechanics, 75, 2575-2581.

[32] Burniston, EE (1969). An Example of a Partially Closed Griffith Crack. International Journal of Rock Mechanics and Mining Sciences, 5, 17-24.

[33] Tweed, J (1970). The deterinination of the stress intensity factor of a partially closed Giffith crack. International Journal of Engineering Sciences, 8, 793-803.

[34] Thresher, RW, Smith, FW (1973). The partially closed Griffith crack. International Journal of Fracture, 9, 33-41.

[35] Theocaris, PS, Pazis, DN, Konstantellos, BD (1986). The exact shape of a deformed internal slant crack undêhiaxial loading. International Journal of Fracture, 30, 135-153.

[36] ASTM (1988). Standard method for splitting tensile strength of intact rock core specimens. 04.48, 471-475.

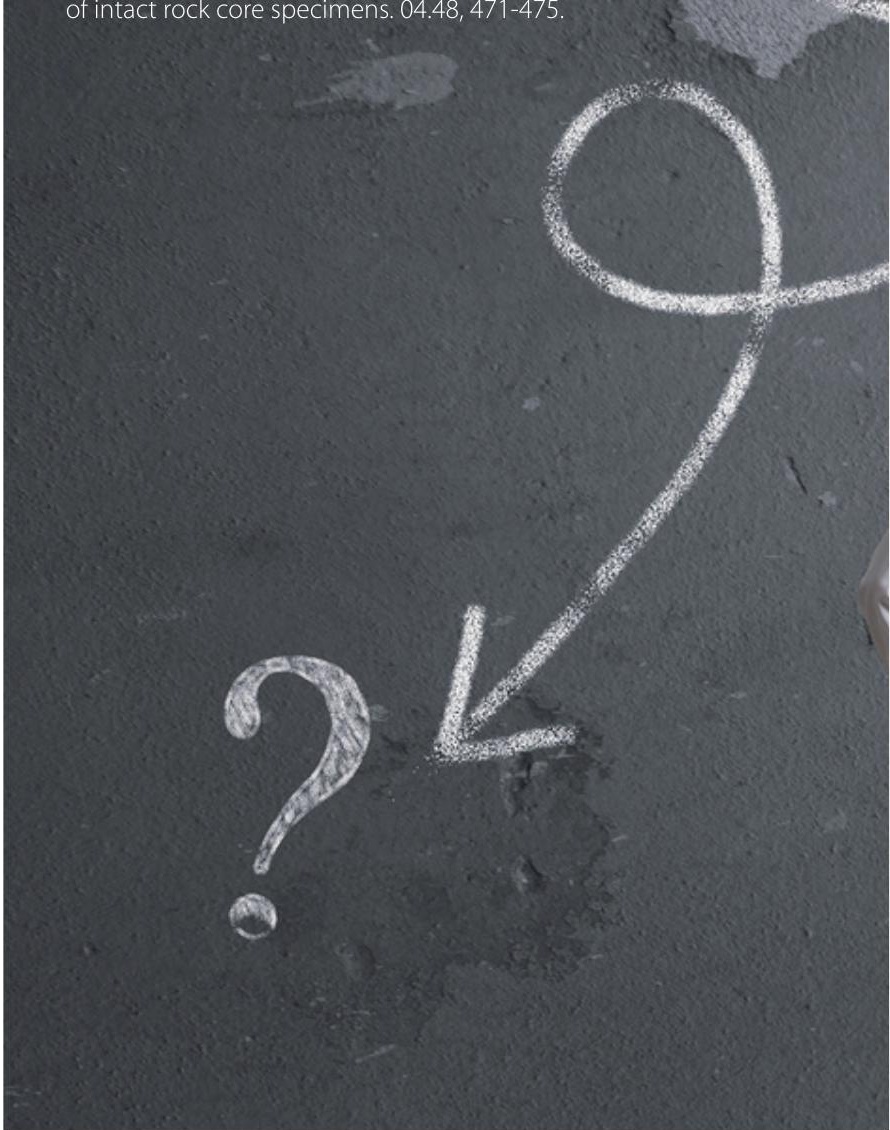




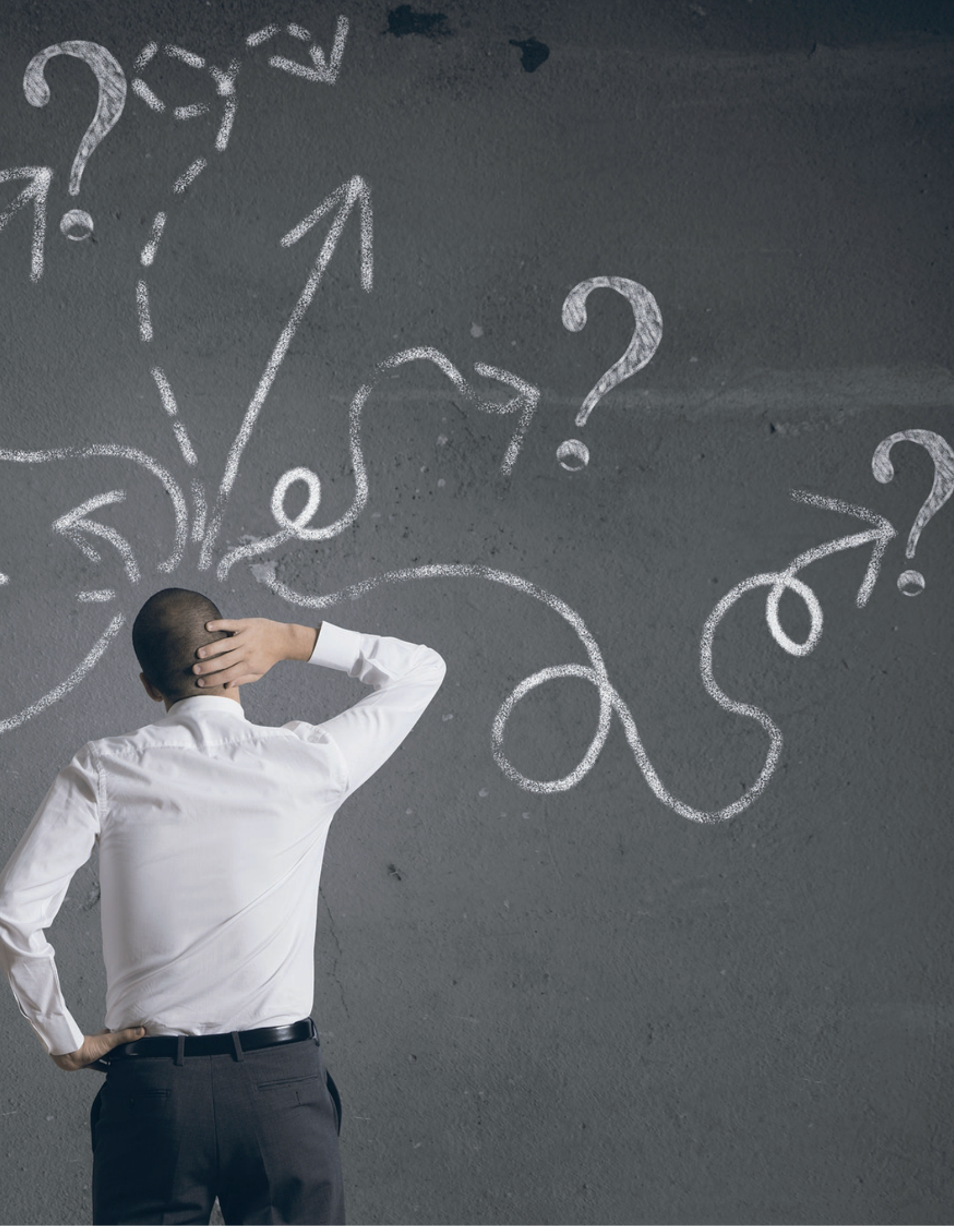

